\title{
La découverte de la circulation sanguine: révolution ou refonte?**
}

Claire Crignon

\section{Summary}

The discovery of the principle of blood circulation by William Harvey is generally considered as one of the major events of the "scientific revolution" of the 17 th century. This paper reconsiders the question by taking in account the way Harvey's discovery was discussed by some contemporary philosophers and physicians, in particular Fontenelle, who insisted on the necessity of redefining methods and principles of medical knowledge, basing themselves on the revival of anatomy and physiology, and of its consequences on the way it permits to think about the human nature. This return allows us to consider the opportunity of substituting the kuhnian scheme of "structure of scientific revolutions" for the bachelardian concept of "refonte".

Keywords: blood circulation; Fontenelle; scientific revolution

\section{Résumé}

La découverte du principe de circulation sanguine par William Harvey est considérée comme un des événements majeurs de la «révolution scientifique» du XVII ${ }^{\mathrm{e}}$ siècle. Cet article reconsidère la question en envisageant la manière dont la découverte de Harvey a été discutée par certains philosophes et médecins contemporains, tout particulièrement Fontenelle. Ce dernier

* Cet article est la version remaniée et écrite d'une conférence prononcée à l'Université de Laval (Québec) ainsi qu'à Mac Gill University (Montréal) en janvier 2009. Je remercie T. Belleguic (et le CIERL) ainsi que F. Charbonneau pour leur invitation. B. Joly, P.-F. Moreau, F. Chareix et M.-F. Pellegrin ont bien voulu relire cet article et me faire part de leurs remarques et suggestions. L'aide de M. S. Seguin a par ailleurs été précieuse pour s'orienter dans la lecture des volumes de l'Académie.

Claire Crignon, Maître de conférence en philosophie à l'Université de Paris-Sorbonne (Paris IV) - ANR JCJC 0145-01, UFR de Philosophie et Sociologie, 1 rue Victor-Cousin, F-75005 Paris (claire.crignon@paris-sorbonne.fr). 
insiste sur la nécessité de redéfinir les méthodes et les principes du savoir médical en tenant compte du renouveau de l'anatomie et de la physiologie, et de ses conséquences sur la manière de penser la nature humaine. Ce retour permet de s'interroger sur l'opportunité de substituer au schéma kuhnien de la «structure des révolutions scientifiques» le concept bachelardien de «refonte».

Mots-clés: circulation sanguine, Fontenelle, révolution scientifique

La découverte du principe de circulation sanguine par William Harvey au début du XVII ${ }^{\mathrm{e}}$ siècle, et son exposition avec la publication de l'Exercitatio anatomica de motu cordis et sanguinis in animalibus (Essai anatomique sur le mouvement du cœur et du sang chez les animaux) à Francfort en 1628, sont considérés comme des événements majeurs dans l'histoire de la médecine moderne. Pourtant, comme le souligne Harold J. Cook dans un article consacré aux liens entre l'essor de la philosophie moderne et les transformations de la médecine au XVII ${ }^{e}$ siècle ${ }^{1}$, l'étude des relations entre la médecine et le mouvement intellectuel dénommé conventionnellement «révolution scientifique»a suscité des dissensions entre historiens des sciences depuis de nombreuses années.

Une première tendance interprétative a consisté à minorer le rôle de la médecine et de la physiologie dans le mouvement de transformation de la science à l'époque moderne. Le sentiment longtemps partagé selon lequel la médecine appartiendrait à la catégorie des «sciences appliquées» a contribué à imposer la conviction que le bouleversement intellectuel qui a lieu entre la fin de la Renaissance et la fin du XVIII ${ }^{e}$ siècle devait être expliqué uniquement à partir des découvertes accomplies dans le domaine des sciences physiques «pures». Du même coup, les découvertes réalisées dans le domaine médical, et en particulier la découverte de la circulation sanguine, n'ont souvent été évaluées qu'en fonction de la manière dont elles permettaient de confirmer l'hypothèse du passage d'un «monde clos à un univers infini» (qui se traduit par le processus de mathématisation de la nature et de géométrisation de l'espace décrit par Alexandre Koyré) ou de la manière dont elles traduisaient l'adoption d'un modèle d'explication mécaniste de la nature (cf. R. S. Westfall) ${ }^{2}$.

Une seconde tendance historiographique s'est efforcée au contraire d'accorder un rôle prépondérant à la médecine de l'âge classique en mettant en

1 Cook 1990, 397-436.

2 Pour la tendance à exclure la médecine du champ de la révolution scientifique on peut renvoyer à Koyré 1957 et à Gillipsie 1960, mais aussi dans ses premiers écrits à Hall 1954, 1963. Hall souligne le rôle de la médecine dans la révolution scientifique à partir de 1983. Pour la tendance à opposer modèle mécaniste et survivances superstitieuses, voir Westfall 1971. 
avant l'héritage de la médecine chimique et de Paracelse ${ }^{3}$ (ou encore celui d'Aristote et de la tradition scolastique) dans la pensée des médecins des débuts de l'époque moderne ${ }^{4}$. La très forte présence du modèle galénique des humeurs et des tempéraments dans le discours médical jusqu'à la fin du XVIII ${ }^{\mathrm{e}}$ siècle serait un signe de l'incapacité du savoir médical à rompre avec les modèles anciens, tout comme les efforts des adeptes de la médecine chimique pour soigner à distance pourraient être lus comme la persistance de croyances magiques et de pratiques occultes 5 .

La reconnaissance du rôle joué par la médecine au sein de la «révolution scientifique» ne date que du début des années 80 au moment où les historiens des sciences font apparaître la présence et l'activité importante des médecins au sein des institutions savantes (Académie Royale des Sciences en France, Royal Society en Angleterre ${ }^{6}$ ). Alors que le succès du modèle kuhnien de la «structure des révolutions scientifiques» ${ }^{7}$ avait pu inciter certains historiens de la médecine à présenter la découverte de Harvey comme un événement «révolutionnaire» ${ }^{8}$ et à interpréter la persistance du modèle galénique des humeurs comme le signe de la difficulté à dépasser un «paradigme» ancien, des voix se sont élevées pour contester la légitimité même du vocable de la «révolution scientifique», particulièrement inadapté dans le cas de la médecine, qui se définit autant comme une science que comme une pratique et n'a pas encore pleinement défini au XVII ${ }^{\mathrm{e}}$ siècle ses normes méthodologiques ${ }^{9}$. Des historiens de la médecine, comme Lester S. King ont montré, dès les années 60 , les limites d'une approche consistant à interpréter la persistance du modèle des humeurs comme un simple «obstacle épistémologique» sur la route du progrès des sciences médicales et souligné qu'on ne pouvait se contenter de comprendre les résistances qui s'expriment au sujet du principe de circulation sanguine (chez William Temple ou Jonathan Swift en Angleterre) comme de simples symptômes d'une attitude conservatrice et hostile par principe à l'affirmation de la nouveauté en science ${ }^{10}$.

Si l'on veut éviter la tentation d'un jugement rétrospectif sur cette découverte et sur les polémiques qu'elle a pu susciter, il apparaît effectivement

3 Debus 1965.

4 Pagel 1967.

5 Boas Hass 1966.

6 Hunter 1982 et 1989.

7 Kuhn 1970 [1962]).

8 Grmek 1990, 91.

9 L'idée de révolution appliquée à la médecine est encore très présente dans French/Wear 1989. Pour une réévaluation de l'idée de «révolution scientifique», voir Osler 2000. Pour une réévaluation critique de cette idée et la mise en évidence de ses limites plus particulièrement dans le domaine des sciences du vivant, voir Cunningham/Perry 1993, 413.

10 King 1965. 
indispensable de commencer par étudier la manière dont elle est présentée et discutée par les contemporains ou les successeurs proches de Harvey. C'est souvent à partir de sa réception cartésienne qu'est évalué l'impact de la découverte du principe de circulation sanguine sur la pensée philosophique à l'époque moderne ${ }^{11}$. Descartes est en effet l'un des premiers à en reconnaître l'importance et à en attribuer la paternité à Harvey ${ }^{12}$. Il convient pourtant, si l'on veut appréhender les répercussions et les enjeux philosophiques de cette découverte, d'engager une étude plus systématique et approfondie des auteurs qui la mentionnent ou s'en font l'écho. Cela implique, premièrement, de s'intéresser à la place qui lui est attribuée au sein de ce que l'on appelle la «révolution scientifique» (relativement aux découvertes réalisées dans les domaines de l'astronomie, avec Copernic et Galilée $\left.{ }^{13}\right)$. Cela suppose, deuxièmement, de regarder de plus près comment les philosophes ou les médecins insistent sur la nécessité de redéfinir les méthodes et les principes du savoir médical en tenant compte de la manière dont le renouveau de l'anatomie et de la physiologie a conduit à penser tout autrement la nature de l'être humain. Lorsque Marchamont Nedham examine, dans un ouvrage publié en 1665 (Medela Medicinae), l'état des sciences et de l'art médical, il prend appui sur la redéfinition de la maladie et sur la remise en cause de la théorie des humeurs pour montrer que ces évènements imposent de trouver une nouvelle méthode pour l'art et la science médicales, une méthode adaptée à la nouvelle constitution de l'être humain («new methods, and rules of curation agreable to new frame of humane nature, and to the new Phaenomena of Diseases» $)^{14}$.

Avant de se poser la question de savoir si les découvertes médicales sont utiles, les auteurs de cette période s'interrogent sur la manière dont elles engagent une redéfinition de la nature de l'être humain, de son statut et de sa place dans l'univers, et du même coup une reconfiguration ou une «refonte» du savoir médical. Si le terme de «révolution» semble inadapté pour caractériser le changement qui s'opère dans le domaine de la science du corps vivant, l'usage du terme de «refonte», que l'on trouve par exemple sous la plume de Fontenelle, paraît plus à même de rendre compte des enjeux de la découverte de la circulation sanguine et de la manière dont la reconfi-

11 Voir sur cette question French 1994, Fuchs 2001, Aucante 2006.

12 La reconnaissance de cette paternité reste largement disputée jusqu'au XVIII ${ }^{\mathrm{e}}$ siècle, en témoigne en particulier l'article «Circulation du sang», Diderot et D'Alembert 1751-1780, vol. 3, 467. Pour Descartes, voir Discours de la Méthode, Euvres, t. VI, 1996, 50-51.

13 Voir par exemple la manière dont Hobbes mentionne Harvey, aux côtés de Copernic et de Galilée, dans l'épître dédicatoire du De Corpore 1999 (1665) 3-4. Henry More consacre au milieu du XVII ${ }^{\mathrm{e}}$ siècle un poème à la découverte de la circulation sanguine, célébrant Harvey aux côtés de Copernic ou de Galilée. Cf. Shugg/Sherwin/Freyman 1979, 180-189.

14 Nedham, 1665, 204. 
guration du savoir physiologique s'enracine dans une redéfinition de la nature de l'être humain.

L'œuvre de Fontenelle est certes moins connue pour son appréciation du savoir médical que pour celle qu'elle propose du savoir mathématique (le calcul infinitésimal en particulier) ou géométrique. Certains textes posent pourtant de manière spécifique la question du statut à accorder au savoir et aux découvertes médicales ainsi que celle du statut du corps humain, objet de la philosophie naturelle. C'est le cas en particulier lorsque Fontenelle évoque, dans les volumes de l'Histoire de l'Académie Royale des Sciences $(1699-1703)^{15}$, la controverse autour de la circulation du sang dans le fœtus. La découverte de Harvey est par ailleurs mentionnée plus directement dans les Nouveaux Dialogues des Morts (1683), lorsque l'auteur s'interroge sur les motifs et les enjeux de la querelle entre partisans de l'ancienne médecine (représentée par Erasistrate) et de la médecine moderne (représenté par William Harvey). Il montre alors que ce qui se joue dans ce combat dépasse de bien loin la question de l'utilité d'une découverte pour le genre humain.

Ce retour aux textes contemporains ou proches du renouveau de l'anatomie et de la physiologie nous permettra in fine de nous interroger sur l'opportunité de substituer au schéma kuhnien de la «structure des révolutions scientifiques» le concept bachelardien de «refonte» utilisé dans la Formation de l'esprit scientifique (1938). Nous voudrions défendre ici l'idée que l'étude de la réception d'une découverte à son époque permet de mieux prendre la mesure de la transformation qui s'opère dans le domaine des sciences médicales. Cette voie interprétative confirmera peut-être l'idée défendue par l'historien des sciences français selon laquelle une «rupture» vis-à-vis des savoirs anciens ne se conçoit jamais isolément mais se transmet à l'intégralité du nouveau savoir qui doit alors trouver un nouveau type de configuration ou d'organisation.

La réflexion de Fontenelle sur les découvertes médicales de son temps peut paraître secondaire ou mineure par rapport à la lecture qu'il propose des découvertes effectuées dans le domaine de l'astronomie, des mathématiques ou de la géométrie, si l'on songe par exemple aux Entretiens sur la pluralité des mondes habités (1686) ou aux écrits sur le calcul infinitésimal $(1727)^{16}$. Nommé secrétaire perpétuel de l'Académie royale des sciences de Paris à partir de son renouvellement en 1699, il le restera jusqu'à sa mort en 1757. Fontenelle a donc été amené à s'intéresser de très près non seulement aux sciences spéculatives, comme les mathématiques et la géométrie, mais aussi aux sciences physiques ou empiriques. Par sciences «physiques», il faut

15 Fontenelle 1699-1703 (désormais HARS).

16 Fontenelle 1686 et 1727, dans: Euvres complètes 1990.

Gesnerus 68 (2011) 
entendre ici au sens large, comme le rappelle Maria Susana Seguin, «non seulement la physique au sens moderne, mais surtout les sciences de la Nature et du vivant» ${ }^{17}$. Fontenelle est amené à s'intéresser à des débats et à des découvertes qui touchent aussi bien la géologie, que la botanique, la zoologie, la chimie, et enfin l'anatomie et la médecine.

Il est en particulier chargé de rédiger une Histoire de l'Académie Royale des Sciences, chaque volume comprenant d'une part les mémoires «écrits par les membres et les correspondants de l'académie», et d'autre part «l'histoire de l'académie des sciences» ${ }^{18}$ rédigée par le secrétaire perpétuel «où est reproduit un résumé, classé par discipline scientifique, des principales interventions faites devant l'académie» ${ }^{19}$. Si Fontenelle accorde une place moins importante aux comptes-rendus portant sur les sciences empiriques qu'à ceux portant sur les sciences spéculatives, les premiers sont pourtant «l'occasion de réflexions plus variées, aussi bien sur la nature du texte scientifique que sur les limites et les possibilités de l'esprit humain» ${ }^{20}$. Parmi les sujets relatifs à la médecine, la question de la circulation du sang dans le fotus occupe une partie importante des comptes-rendus rédigés par Fontenelle depuis le début du XVIII' siècle jusqu'aux alentours de $1725^{21}$.

$\mathrm{Au}$ moment où Fontenelle écrit sur ces sujets, le consensus autour de la découverte de la circulation sanguine s'est établi. Depuis la création en 1673 par Louis XIV d'une chaire d'anatomie au jardin des plantes, la bataille de la circulation paraît gagnée en France ${ }^{22}$. Dans le premier volume de l'Histoire de l'Académie Royale des Sciences (1699), Fontenelle peut présenter le principe de circulation sanguine comme un fait que nul ne peut ignorer:

17 Seguin 2010. On peut rappeler la définition du médecin que donne Christopher Merrett, médecin et physiologiste proche de Harvey: «The word Physician, derived from the Greek 〈phusikos〉, is plainly and fully rendered by the word Naturalist, (that is) one well vers'd in the full extent of Nature, and Natural things [...].» Merrett 1660, 2-3.

18 «Cette Histoire contient deux parties. L'une que l'on a voulu appeler plus particulièrement Histoire, est l'Extrait \& l'Abrégé de tout ce qui s'est dit de remarquable dans l'Académie, soit par écrit, soit de vive voix. L'autre Partie, ce sont les Mémoires, c'est à dire, celles d'entre toutes les Pièces lûes dans l'Académie, qui ont été jugées les plus importantes, \& les plus dignes d'être données au public dans toute leur étenduë.». Fontenelle 1699, Préface, II.

19 Académie des Sciences 1996,114. Il est à noter que le mémoire n'est pas toujours donné à la suite de l'histoire pour chaque année. On trouve aussi à la fin de la partie histoire, les éloges des membres décédés.

20 Seguin 2006, 151.

21 Voir par exemple les années 1699,1701,1703,1720, 1725.

22 «Si l'Anatomie a beaucoup d'obligation à Harvée qui a découvert à la Circulation, à Virsungus qui a trouvé le canal Pancréatique, à Asellius qui a fait voir les vénes lactées, à Pecquet qui le premier a démontré le canal thorachique, \& à plusieurs Modernes qui y ont travaillé avec succès, elle n'en a pas point à Monsieur Daquin premier Medecin du Roy, par le rétablissement qu'il fit des Démonstrations publiques au Jardin royal, où il a voulu que l'Anatomie fut démontrée suivant la Circulation du sang, \& les dernières découvertes.» Dionis 1690, Préface. 
«Personne n'ignore que le sang de tout le corps, rapporté au cœur par la veine cave tombe dans l'oreillette droite, par-delà le ventricule droit, d'où le cœur en se resserrant le pousse par l'artère pulmonaire dans le poumon. Les veines du poumon le reprennent, le portent dans l'oreillette gauche du cœur, d'où il tombe dans le ventricule gauche, qui par sa contraction le pousse ensuite dans l'aorte, \& le répand dans toutes les artères du corps, après quoi les veines le reprennent, \& le rapportent dans la veine cave. C'est là ce qu'on appelle la circulation du sang.» ${ }^{23}$

Quelques années plus tôt, en 1683, dans le $5^{\mathrm{e}}$ des Nouveaux Dialogues des Morts, Fontenelle imaginait la rencontre entre le représentant de la médecine moderne, William Harvey, et Erasistrate, représentant de l'ancienne tradition médicale. Répondant à l'étonnement du médecin de l'école d'Alexandrie, le personnage de Harvey mis en scène par Fontenelle répondait: «J'en ai fait voir tant d'expériences, que personne n'en doutera plus. $\rangle^{24}$. L'accent est mis dans ce texte, comme dans la Préface de l'Histoire de l'Académie Royale des Sciences, moins sur la nouveauté de la découverte, que sur la manière dont Harvey a pu apporter des preuves expérimentales du principe de circulation sanguine: «En Angleterre [...] Harvé [s'est rendu célèbre] par la découverte, ou du moins par les preuves incontestables de la circulation du sang. ${ }^{25}$

S'il s'agit donc d'un fait avéré dont personne ne peut plus douter, pourquoi Fontenelle s'y arrête-t-il cependant? D'abord parce qu'il lui importe de préciser le statut des découvertes réalisées dans le domaine des sciences du vivant (ce que Fontenelle désigne par le terme de «Physique») par rapport à celles qui ont pu être accomplies dans les domaines des sciences spéculatives, comme les mathématiques ou la géométrie. Dans la préface du premier volume de l'Histoire de l'Académie Royale des Sciences, Fontenelle fait remarquer que si nous «avons lieu de nous promettre que le progrès des sciences sera heureux», si l'on «s'est mis sur les bonnes voies [que] depuis environ un siècle», «nous sommes obligés à ne regarder présentement les Sciences que comme étant au berceau, du moins la Physique» ${ }^{26}$. Comme le souligne M. S. Seguin, ce retard des sciences du vivant par rapport aux mathématiques ou à la géométrie s'explique par la nature particulière des objets sur lesquels elles portent: le fonctionnement du corps humain, «l'origine de la vie» (la formation du foetus et la circulation du sang dans le fœtus). Il ne s'agit pas ici de tenir un discours sur les idées mais «d'élaborer des idées à partir des faits, et donc à partir du monde sensible». Voilà pour-

23 Anatomie, «De la circulation du sang dans le fœetus», HARS 1699, 25.

24 Fontenelle 1990, t. 1, 93.

25 Fontenelle 1996, 338.

26 HARS, 1699, Préface, XVIII. 
quoi, même si le phénomène de la circulation sanguine s'impose comme un fait prouvé expérimentalement, il n'en demeure pas moins nécessaire de tenir compte de la nature particulière de l'objet à connaître - le corps humain dans sa matérialité - et du fait qu'il s'agit de rendre compte du principe même de la vie. Le «langage mathématique», l'esprit géométrique que Fontenelle propose de transporter à d'autres connaissance ${ }^{27}$ se trouve donc «mis à l'épreuve de l'explication de la Nature» ${ }^{28}$.

L'intérêt de la démarche de Fontenelle dans les volumes de l'Histoire de l'Académie Royale des Sciences réside précisément dans la manière dont il rend compte du progrès des sciences médicales, en donnant aux sciences du vivant un statut bien spécifique et en montrant que les progrès réalisés dans les domaines de la physiologie ou de la médecine ne peuvent sans doute pas être évalués à l'aune des seuls critères de la mathématisation et de la mécanisation de la nature.

La volonté de rendre compte du mécanisme de la circulation sanguine (l'image du cœur comme pompe) ne doit en effet pas nous faire perdre de vue le caractère «merveilleux» du corps humain. La «merveille», comme le souligne M. S. Seguin, n'est pas ce qui suscite «l'admiration stérile», ni «la présence d'un être transcendant», mais ce qui vient limiter les connaissances humaines ou ce qui, en dépit de toutes les explications apportées, doit continuer à susciter notre curiosité 29 . La «merveille» désigne une réalité «que l'esprit humain n'a pas encore pu élucider» ou en tous les cas pas totalement ${ }^{30}$.

Or, précisément ce vocable du merveilleux intervient dans le cinquième des Nouveaux Dialogues des Morts - au tout début du texte - lorsqu'Erasistrate s'adresse de manière ironique à Harvey:

«Vous m'apprenez des choses merveilleuses. Quoi, le sang circule dans le corps, les veines le portent des extrémités au cœur, et il sort du cœur pour entrer dans les artères, qui le reportent vers les extrémités!».

Certes on peut choisir de ne voir dans cette apostrophe qu'une manière d'ironiser sur la prétendue découverte de Harvey, l'une des tactiques les plus courantes de ses adversaires consistant à minimiser sa découverte en montrant que ce principe était déjà connu de ses prédécesseurs. Mais on peut aussi la comprendre comme une manière pour Fontenelle d'indiquer d'emblée que les découvertes de la médecine moderne au sujet du corps humain ne doivent pas faire perdre de vue son statut particulier de «merveille». Ce qui implique que l'explication du mécanisme de la circulation sanguine qui

27 Fontenelle 1994, 44.

28 Seguin 2010, 4.

29 Seguin 2010, 6.

30 Seguin 2006, 156-57. 
incombe au médecin ne peut jamais épuiser la tâche de compréhension qui incombe au philosophe.

L'insistance posée sur le caractère moins «révolutionnaire» ${ }^{31}$ de cette découverte que sur son aspect «merveilleux» $»^{32}$ indique aussi que le savoir médical dont on peut disposer, une fois acquise la découverte de Harvey, n'est pas clos. «Tant de choses qui restent encore, et dont apparemment plusieurs resteront toujours à savoir, donnent lieu au découragement affecté de ceux qui ne veulent pas entrer dans les épines de la Physique», écrit Fontenelle dans la Préface sur l'utilité des Mathématiques et de la Physique et sur les travaux de l'Académie des sciences ${ }^{33}$. Contre cette tendance, Fontenelle suggère que l'absence de clôture de notre connaissance du monde physique, la manière dont la Nature vient mettre à l'épreuve notre faculté de connaître et rendre sensibles ses limites, devraient au contraire nous inciter à continuer les observations et les expériences. Celles-ci, à force d'être répétées, permettront peut-être à la connaissance du monde physique de progresser: «La nature [...] n'est jamais si admirable, ni si admirée que quand elle est connue.» ${ }^{34}$.

Et c'est bien dans cette voie qu'il s'engage, dans les premiers volumes de l'HARS, lorsqu'il rend compte des disputes qui opposent les médecins de son temps au sujet du principe de circulation du sang chez le fœtus. Ce débat occupe l'Académie Royale des Sciences dès son renouvellement en 1699: «A peine l'Académie avait-elle pris la nouvelle forme que le Règlement lui donnait, qu'elle fut occupée de cette contestation ${ }^{35}$. L'enjeu est ici de taille

31 «Tout en enregistrant de multiples transformations en profondeur et d'inépuisables capacités innovatrices dans tous les champs disciplinaires, la seule révolution que Fontenelle ait jamais signalée est une révolution étroitement «régionale〉 et circonscrite au champ des mathématiques. Fontenelle n'a en effet jamais employé le vocable de <révolution〉 hors de ce domaine et, a fortiori, n'a jamais généralisé son usage: il a donc ignoré le syntagme «révolutions scientifique >.» Mazauric 2007, 219.

32 L'adjectif «merveilleux» appliqué aux découvertes médicales de l'époque moderne est aussi utilisé par Jean Bernier dans l'une des premières histoires de la médecine parue en 1689, en un sens beaucoup plus trivial cependant que chez Fontenelle. Son emploi résulte là aussi de la fiction d'un retour des médecins antiques à l'époque moderne: «Il ne faut donc pas douter que si Hippocrate et les grands hommes qui l'ont suivi revenaient au monde, ils ne fussent surpris et étonnés de voir les merveilles qu'on a découvertes depuis eux dans la théorie et la pratique de la Médecine, et même le jour que tant de doctes plumes ont donné à leurs écrits.» Bernier 1689, I, 2, 11.

33 Fontenelle 1994, 47.

34 HARS 1699, Préface, XVI.

35 HARS 1699, 29. Voir aussi l'éloge de M. Méry: «Mais ce qui a fait le plus de bruit dans ses Volumes, a été son opinion sur la circulation du sang dans le fœetus, ou sur l'usage du trou ovale, directement opposée à celle de tous les autres anatomistes. Il fut cause que l'Académie, dès son renouvellement en 1699, fut agitée par cette question. Un monde d'Adversaires élevés contre lui, tant au dedans qu'au dehors de l'Académie, ne l'ébranla point [...]. On ne sait point encore aujourd'hui quel parti est victorieux, et c'est une assez grande gloire pour celui qui seul était un parti. Il paraît, ainsi que nous osâmes le soupçonner il y a longtemps, que les deux systèmes opposés pourraient être vrais, et se concilier [...]». Fontenelle 1996, 14. 
car ce dont il s'agit de rendre compte, c'est bien de l'origine même de la vie. L'explication proposée par Harvey et par Richard Lower, suivie par «tous les Anatomistes» paraissait, écrit Fontenelle, si «conforme à l'état et aux besoins du fœtus, que l'on se tenoit sûr d'avoir découvert sur cela le secret de la Nature» ${ }^{36}$. Mais les recherches menées par Jean Méry, médecin anatomiste et chirurgien de l'Académie Royale des Sciences, vont faire apparaître que «les parties qui servent à la circulation ne sont pas tout à fait les mêmes dans le foetus» que dans le cœur de l'être vivant après la naissance. Dans le fœtus, «la cloison qui sépare les deux oreillettes du cœur est percée d'un trou qu'on appelle le trou ovale», ce qui permet au sang de circuler dans le fœtus avant que les poumons ne se développent. Une fois le fotus né, «le trou ovale se ferme peu à peu, et le canal de communication se dessèche» ${ }^{37}$. Deux médecins s'opposent à la thèse défendue par Méry: Joseph-Guichard Du Verney et son élève Tauvry ${ }^{38}$.

L'intérêt du compte-rendu que propose Fontenelle tient sans doute moins au contenu des explications proposées au sujet de la circulation sanguine dans le fœtus, qu'à la manière dont il en fait un prétexte pour mettre en évidence les difficultés propres à la connaissance du corps humain. Pour trancher la dispute, que Fontenelle qualifie de «guerre anatomique»" ${ }^{39}$, l'Académie choisit dans un premier temps de s'en rapporter aux faits. L'observation anatomique devrait pouvoir nous dire si c'est l'artère pulmonaire ou l'aorte qui reçoit le plus de sang, et cela en fonction de «la grosseur de l'aorte et de l'artère pulmonaire dans le fœtus». On nomme donc «des commissionnaires pour voir exactement les faits que l'on produirait de part et d'autre». Cette investigation impartiale se traduit cependant par un échec puisque chaque parti produit une observation empirique qui confirme sa théorie: «M. Méry fit voir [...] l'artère pulmonaire plus grosse que l'aorte, et M. Tauvry plus petite», «tant il est vrai», souligne Fontenelle, «qu'en matière de Physique les simples questions de fait, qui ne sont cependant que préliminaires, ont souvent elles-mêmes beaucoup de difficulté» ${ }^{40}$.

Si ce type d'observation empirique ne suffit pas, c'est peut-être d'abord parce qu'elle se limite à appréhender le principe de circulation sanguine

36 «Physique particulière, anatomie, de la circulation du sang dans le fœetus», HARS 1699, 26.

37 HARS 1699, 25. Voir aussi Diderot et D'Alembert, article «Circulation du sang», Encyclopédie ou Dictionnaire raisonné des arts, des sciences ou des métiers, vol. III, 1988.

38 Jean Méry, né en 1645, entre à l'Académie Royale des Sciences en 1684, y reste jusqu'en 1722, il découvre la fonction du trou ovale. Cf. Salomon-Bayet 2008, 73; voir aussi «Eloge de Monsieur Méry» par Fontenelle1996, 9-17. Joseph-Guichard Du Verney (1648-1730), lègue ses pièces anatomiques à l'Académie en 1730, voir Académie des Sciences 1996, 258. Sur Du Verney, voir l'éloge de Fontenelle 1996, 189-198.

39 «Sur l'histoire du fœtus», HARS 1699, 34.

40 HARS 1699, 29. 
dans le foetus d'un point de vue purement mécaniste. Or, comme le souligne Fontenelle dans la suite du texte $e^{41}$, ce premier niveau de compréhension des phénomènes, certes nécessaire, n'est cependant pas suffisant. Il faut toujours rechercher «les raisons des structures mécaniques». Il faut tenter de comprendre «le plan, l'esprit général» des «productions particulières de la nature», en se demandant par exemple ce qui rend compte de l'existence de deux types de circulations, la petite (distribution du sang par les veines pulmonaires) et la grande (distribution du sang par les veines au corps tout entier $)^{42}$. La mécanisation de la nature, qu'elle concerne l'univers ou le corps humain, ne doit pas occulter le merveilleux ou le sublime qui s'attachent à ces objets, comme le faisait déjà remarquer à la Marquise le philosophe des Entretiens sur la pluralité des mondes ${ }^{43}$. Le recours à «l'anatomie comparée», autrement dit à un acte de comparaison propre à l'esprit humain, permet de dépasser la simple observation des faits, pour appréhender la complexité du phénomène de circulation sanguine à partir de la diversité des ouvrages de la Nature. Il permet aussi de contourner une des difficultés propres à la science du vivant en rendant sensible et manifeste, ce qui demeure «confus» ou «invisible» à travers l'observation du corps humain ${ }^{44}$.

Si les faits ne permettent pas de trancher entre partisans de l'explication traditionnelle (Harvey, Lower et la plupart des anatomistes) et celle de Méry, c'est peut-être ensuite parce qu'il n'y a pas «d'impossibilité absolue» entre les deux explications. Il est possible que «la nature [mette] en usage ces deux différents moyens en différentes espèces d'animaux» ou qu'elle «les

41 HARS 1699, 31.

42 «L'homme, et la plus grande partie des animaux, destinés à beaucoup de mouvements divers, et à des fonctions d'une grande vivacité, doivent avoir un sang tout pénétré de particules aériennes, et c'est pour cela qu'il se fait en eux deux circulations différentes, l'une de toute la masse du sang par le poumon, afin qu'elle aille prendre à chaque instant dans ce réservoir rempli d'un air toujours nouveau, tout ce dont elle a besoin, l'autre de cette même masse chargée d'air par tout le reste du corps, où elle va se répandre avec les qualités salutaires qu'elle a acquises dans le poumon.» HARS 1699, 34-35.

43 On peut appliquer au mécanisme de la circulation du sang la réponse qui est faite à la Marquise au sujet des explications de l'univers proposées par Descartes et les Modernes: «A ce compte, dit la Marquise, la philosophie est devenue bien mécanique? Si mécanique, répondis-je, que je crains qu'on en ait bientôt honte. On veut que l'univers ne soit en grand, que ce qu'une montre soit en petit, et que tout s'y conduise par des mouvements réglés qui dépendent de l'arrangement des parties. Avouez la vérité. N'avez-vous pas eu quelquefois une idée plus sublime de l'univers [...]?» Fontenelle 1990, t. 2, 21.

44 «L'anatomie des animaux nous devrait être assez indifférente, il n'y a que le corps humain qu'il nous importe de connaître. Mais telle partie dont la structure est dans le corps humain si délicate ou si confuse qu'elle en est invisible, est sensible et manifeste dans le corps d'un certain animal. De là vient que les monstres mêmes ne sont pas à négliger. La Mécanique cachée dans une certaine espèce ou dans une structure commune se développe dans une autre espèce, ou dans une structure extraordinaire, et l'on dirait presque que la Nature à force de multiplier et de varier ses ouvrages ne peut s'empêcher de trahir quelquefois son secret.» HARS 1699, Préface, X. 
emploie indifféremment dans la même espèce», comme le suggèrera finalement Fontenelle dans le volume de $1703^{45}$ et comme il le répètera dans son éloge de Monsieur Méry: «Il paraît, [...] que les deux systèmes opposés pourraient être vrais et se concilier [...].» ${ }^{46}$

Ainsi l'objectif de la connaissance médicale du corps humain n'est pas tant de percer les secrets de la nature ou de montrer la simplicité de ses voies que de dévoiler la complexité des principes qui rendent compte de la vie, ce qui conduit souvent Fontenelle à adopter une position sceptique au sujet des sciences de la nature. La «guerre anatomique» qui oppose les partisans des explications de Harvey à Méry et aux partisans de son Traité Physique révèle que l'intérêt d'une découverte médicale tient moins à ce qu'elle permet de prouver, qu'à la manière dont elle engage l'esprit humain à rester curieux, à dévoiler ainsi le caractère merveilleux de la structure du corps humain ainsi que les limites de notre connaissance ${ }^{47}$. C'est aussi sans doute ce qui explique que Fontenelle ne cherche pas à donner raison à l'un des camps en présence contre l'autre ${ }^{48}$, mais qu'il tente plutôt de comprendre les raisons mêmes de ce conflit et de sa durée, l'existence de cette dispute reflétant à la fois l'étendue des sujets qui restent à discuter une fois qu'une découverte a été faite et la complexité même de l'objet de la philosophie naturelle (Physique) ou son caractère «épineux», pour reprendre ses propres termes.

45 «Il paraîtrait assez étrange que les deux systèmes contraires pussent être vrais en même temps; cependant il n'y a peut-être pas d'impossibilité absolue. On prétend que dans le veau et dans l'agneau fœtus, les vaisseaux du côté gauche surpassent aussi constamment en capacité ceux du droit, et que dans le fœetus humain ceux du droit surpassent ceux du gauche. Si le fait est bien vrai, M. Méry convient que dans le veau et dans l'agneau fœtus, la circulation se fera selon l'ancien système, et dans le fœtus humain selon le sien. Or si la nature met en usage ces ceux différents moyens en différentes espèces d'animaux, peut-être les emploiet-elle indifféremment dans la même espèce $[. .$.$] .» «Sur la circulation du sang dans le fotus»,$ HARS 1703, 35 .

46 «Il paraît, ainsi que nous osâmes le soupçonner il y a longtemps, que les deux systèmes opposés pourraient être vrais, et se concilier; dénouement qui mériterait d'être remarqué dans l'Histoire de la Philosophie, et qui condamnerait bien la grande chaleur de toute cette contestation.» Fontenelle 1996, 14.

47 Le vocabulaire du merveilleux revient encore sous la plume de Fontenelle, lorsqu'il fait état d'une dissection pratiquée par M. Littré sur un homme de 50 ans mort subitement après avoir pris une «médecine de précaution». L'égalité de la «force de ressort» des deux «troncs de l'aorte» constitue, écrit Fontenelle «une merveille presque incompréhensible» tant il est difficile de concevoir une «égalité si juste exécutée sur un si prodigieux nombre de tuyaux tous différents». «Sur une autre mort subite après une médecine de précaution», HARS 1701, 33. L'observation produite par la dissection permet d'apprécier à nouveau la complexité du phénomène de circulation sanguine.

48 «En cas que la nature fit prendre au sang tantôt une route, tantôt l'autre; quand même l'une des deux serait la plus communément usitée, il n'y aurait pas lieu de s'étonner que cette question eut longtemps partagé des Anatomistes, \& eût fourni aux deux partis des armes assez égales.». «Sur la circulation du sang dans le fœetus», HARS 1703, 36. 
On remarquera au passage que Fontenelle ne mentionne jamais, pour faire état de cette dispute, le critère de l'utilité thérapeutique. Il n'est pas ici question de déterminer si l'une des deux hypothèses en présence peut être considérée comme supérieure à l'autre du point de vue des avancées thérapeutiques qu'elle rendrait possible, alors même que l'absence de respiration du fotus avant la naissance permet de souligner les dangers d'un accouchement par le siège ${ }^{49}$. L'enjeu de cette dispute est ailleurs: il est bien, comme pour tout ce qui a trait à la Physique dans le sens large de ce terme, dans la connaissance du corps humain: «[...] il n'y a que le corps humain qu'il nous importe de connaître», écrit Fontenelle dans la Préface de l'HARS. Mais précisément, on ne peut s'engager dans ce projet de connaissance du corps humain, on ne peut espérer faire des découvertes importantes dans ce domaine et en retirer des bénéfices pratiques, que si l'on accepte aussi de passer du temps à pratiquer des observations, des comparaisons, qui peuvent au premier abord paraître inutiles, comme celles qui relèvent de l'anatomie comparée ${ }^{50}$.

«Telle est la destinée des sciences maniées par un petit nombre de personnes: l'utilité de leurs progrès est invisible à la plupart du monde, surtout si elles se renferment dans des professions peu éclatantes», écrit Fontenelle dans la Préface de l'HARS ${ }^{51}$. Cette remarque qui concerne aussi bien la physique que les mathématiques, traduit bien la situation particulière des sciences médicales dans le courant du XVII ${ }^{\mathrm{e}}$ siècle. Si Fontenelle rappelle dans la Préface sur l'utilité des Mathématiques et de la Physique que c'est à l'aune du critère de l'utilité que l'on évalue généralement les progrès réalisés dans le domaine de la physique, rien n'étant «plus utile» aux hommes pris dans leur «état naturel» «que ce qui peut leur conserver la vie», il souligne aussi la difficulté à rendre manifeste cette utilité: «La détermination des Longitudes par les satellites, la découverte du Canal Torachique, un Niveau plus commode et plus juste, ne sont pas des nouveautés aussi propres à faire du bruit, qu'un Poème agréable, ou un beau discours d'éloquence. ${ }^{52}$

En ce sens, il faut selon Fontenelle accorder la plus grande importance à la manière dont une découverte est défendue, expliquée et communiquée, d'une part au sein d'un public savant comme l'est l'Académie Royale des Sciences, mais aussi d'autre part auprès d'un public plus large. On peut pour s'en convaincre, se référer à la lecture comparée des éloges des deux médecins concernés par la dispute sur la circulation du sang dans le fœtus,

49 Salomon-Bayet 2008, 134.

50 HARS 1699, Préface, X.

51 HARS 1699, Préface, V.

52 Fontenelle 1994, 38-39. 
rédigés par Fontenelle: l'éloge de Méry et celui de Du Verney. Tout, selon lui, oppose ces deux personnages:

«Ils n'avaient rien de commun qu'une extrême passion pour la science, et beaucoup de capacité; du reste presqu'entièrement opposés, surtout à l'égard des talents extérieurs. ${ }^{53}$

Si à première vue, l'avantage semble du côté de Méry, présenté comme un partisan de l'observation et de «l'exactitude des faits», alors que Du Verney se caractérise par sa tendance à «tourner les faits selon ses idées», Fontenelle présente néanmoins Méry comme un savant peu apte à communiquer ses idées, incapable d'apporter les «ménagements nécessaires dans [une] dispute», ou de «quitter facilement ses opinions particulières». Menant une vie retirée et solitaire dans son cabinet d'anatomie, il préférait, nous dit Fontenelle, la compagnie des cadavres qu'il dérobait aux chirurgiens pour les disséquer la nuit «en grand secret» plutôt que celle de ses confrères. A l'opposé, Du Verney est loué non seulement pour ses connaissances mais pour ses dons d'éloquence et sa capacité à communiquer l'enthousiasme propre à une découverte médicale:

«Il n'eût pu annoncer indifféremment la découverte d'un vaisseau, ou un nouvel usage d'une partie; ses yeux en brillaient de joie, et toute sa personne s'animait. Cette chaleur ou se communique aux auditeurs, ou du moins les préserve d'une langueur involontaire qui aurait pu les gagner.. ${ }^{54}$

C'est finalement moins du point de vue du contenu d'une découverte que l'on peut trancher une dispute entre deux savants, que du point de vue de leur capacité à la communiquer et à en faire une nouveauté «aussi propre à faire du bruit qu'un poème agréable». Quant au texte des Nouveaux Dialogues des Morts, il permet à Fontenelle de préciser la manière dont il est possible de rendre compte d'une découverte médicale auprès d'un public pas nécessairement savant mais néanmoins cultivé, comme l'est par exemple la Marquise des Entretiens. Le recours au genre littéraire classique (inauguré par Lucien) des dialogues entre anciens et modernes lui permet de construire la fiction d'une rencontre entre médecine ancienne et médecine moderne. C'est ici le médecin et anatomiste grec Erasistrate, resté célèbre pour sa guérison spectaculaire du fils du roi Syrien Séleucos qui dialogue avec le médecin anglais William Harvey, dont la découverte apparaît comme le point de départ d'une remise en question des fondements sur lesquels la science et la pratique médicale se sont édifiés depuis l'antiquité.

53 «Eloge de M. Du Verney», Fontenelle 1996, 194.

54 Fontenelle 1996, 189. 
De quoi s'agit-il de rendre compte par le recours à cette fiction? Pas vraiment d'une prétendue supériorité des modernes sur les anciens ${ }^{55}$. La fonction du parallèle établi dans ce dialogue entre médecine ancienne et médecine moderne n'est pas de trancher en faveur d'un camp ou d'un autre: «L'équilibre des forces en présence se maintient toujours dans le débat.» ${ }^{56}$ Il s'agit plutôt de se prononcer sur la nature du critère à l'aune duquel il est possible d'évaluer l'importance des découvertes médicales. Or sur cette question, la position de Fontenelle reste très cohérente entre ce texte écrit en 1683 et le texte ultérieur de la Préface sur l'utilité des Mathématiques et de la Physique (1708). Dans le texte de la Préface, Fontenelle explique en effet que l'utilité d'une découverte dans le domaine des sciences apparaît incontestable aux yeux de celui qui est à même de comparer le savoir et la pratique des anciens et ceux des modernes, de celui qui a suffisamment de recul sur les spéculations et les recherches du monde savant pour en apprécier les possibles applications ou usages (ce qui est le cas de Fontenelle qui rédige les comptes-rendus des séances de l'Académie pendant une très longue période). Il n'en va pas nécessairement de même pour le public qui préfèrera «jouir des avantages qu' [une découverte] lui procurera que de les connâ̂tre». Peu importe au malade que le chirurgien qui l'opère soit partisan de l'ancienne médecine ou de la médecine moderne. Ce qui compte, c'est uniquement le succès de l'opération:

«L'anatomie que l'on étudie depuis quelque temps avec tant de soins, n'a pu devenir plus exacte sans rendre la Chirurgie plus sûre dans ses opérations. Les chirurgiens le savent, mais ceux qui profitent de leur Art n'en savent rien. Et comment le sauraient-ils? Il faudrait qu'ils comparassent l'ancienne chirurgie avec la moderne. Ce serait une grande étude qui ne leur convient pas. L'opération a réussi, c'en est assez, il n'importe guère de savoir si dans un autre siècle elle aurait réussi de même.» ${ }^{57}$

La tâche de celui qui rend compte du progrès des sciences est donc double. Il s'agit premièrement de montrer que les progrès accomplis dans le domaine de la philosophie naturelle et dans l'explication des causes des phénomènes, loin de mettre un terme au sentiment du merveilleux ou du sublime, contribuent à les susciter (c'est là le sens du travail accompli par Fontenelle dans l'HARS). Il s'agit aussi deuxièmement, et c'est ce que Fontenelle fait dans ce

55 Le choix d'Erasistrate de Céos (III ${ }^{\mathrm{e}}$ siècle avant J.-Chr.) est à cet égard significatif puisque ce médecin de l'école d'Alexandrie est l'un des premiers à refuser la théorie des humeurs et à donner au cœur une position centrale dans le réseau des veines et des artères (même si les artères véhiculent de l'air selon lui). Contrairement à Galien, ce médecin apparaît plutôt comme un «précurseur» de la médecine moderne.

56 Charbonneau 2006, 68.

57 Fontenelle 1994, 39. 
dialogue, de tenir compte de ce qui constitue un frein à cette appréciation des découvertes médicales, et du fait que la profession médicale fait précisément partie de ces «professions peu éclatantes» où le «public ne jouit du succès qu'elle a eu qu'avec une espèce d'ingratitude» ${ }^{58}$.

On ne doit ainsi peut-être pas distinguer deux types d'écritures de la science médicale chez Fontenelle: une «écriture privée», dont les Nouveaux Dialogues des Morts (1683) rendraient compte et une «écriture institutionnelle» dont témoigneraient les volumes de l'HARS (à partir de 1699), comme le propose Simone Mazauric dans son ouvrage sur Fontenelle et l'invention de l'histoire des sciences à l'aube des Lumières. Il s'agit en effet pour Fontenelle non pas d'écrire la science médicale, mais de la rapporter de deux manières différentes, en visant des publics distincts. Ainsi lorsqu'il rend compte des débats des savants sur la circulation du sang dans le fœtus, il se tient au plus près du discours des médecins, et de leur capacité à communiquer leurs découvertes de manière éloquente. Et lorsqu'il rend compte de ce qui apparaît aux yeux d'un public plus large comme la découverte majeure de la médecine et de la physiologie du XVII ${ }^{e}$ siècle, il fait état de ce qui peut constituer un frein à la reconnaissance d'une découverte: la tendance à n'évaluer une découverte qu'en fonction du seul critère de l'utilité thérapeutique.

Les propos qu'il prête au médecin Erasistrate (resté justement célèbre pour sa guérison) ne font que reprendre les arguments des médecins traditionalistes qui forment encore à la fin du XVII e et au début du XVIII ${ }^{\mathrm{e}}$ siècle «la majorité de la corporation médicale et des enseignants universitaires» ${ }^{59}$. S'il y a autant de morts au siècle de Harvey qu'auparavant, si la «circulation du sang, ces conduits, ces canaux, ces réservoirs», si «tout cela ne guérit [...] rien», alors ne vaut-il pas mieux se fier aux pratiques des anciens? C'est bien à l'aune de ce critère de l'utilité thérapeutique que la découverte de Harvey a effectivement été évaluée tout au long du XVII ${ }^{\mathrm{e}}$ siècle. Non seulement les médecins continuent à pratiquer la saignée jusqu'à la fin du XVIII ${ }^{\mathrm{e}}$ siècle, et ceux qui refusent de le faire sont souvent taxés d'inhumanité ${ }^{60}$, mais en outre les premières tentatives de transfusion sanguines pratiquées par Richard Lower et rapportées dans le Journal des Savants se soldent par «des

58 Fontenelle 1994, 38.

59 Mazzolini 1997, 97.

60 Jean Bernier, dans ses Essais de Médecine parus à Paris en 1689, évoque le refus de la saignée dans un chapitre consacré aux «bizarreries et singularités des médecins» et l'interprète comme un signe d'inhumanité dans la relation du médecin à son patient: «Un autre [médecin] [...] croit se bien distinguer des saigneurs et des Médecins d'eau douce, laissant plutôt brûler le malade vif, ou crever de douleur et de plénitude, que de lui ordonner la moindre saignée [...].» Bernier 1689, II, 13, 381. 
échecs retentissants» ${ }^{61}$. Avant le renouvellement de l'Académie, l'ancienne Académie s'était d'ailleurs engagée (en 1667) dans ces essais de transfusions. Claire Salomon-Bayet rappelle comment le résultat désastreux du passage de l'expérimentation animale à l'expérimentation humaine conduisit le parlement de Paris à «interdire la transfusion par arrêt comme un remède inutile et dangereux» ${ }^{62}$.

En faisant dire à Erasistrate que la découverte de Harvey et celles des anatomistes n'empêcheront pas les hommes de mouririr ${ }^{63}$, Fontenelle ne prend donc pas, comme le suggère $\mathrm{S}$. Mazauric, une position ambiguë, dans le débat entre partisans de la médecine ancienne et de la médecine moderne (position qu'il clarifierait en 1688 avec le Parallèle des anciens et des modernes, puis dans l'HARS, sous l'influence de Claude Perrault $\left.{ }^{64}\right)$. Il souligne simplement l'échec des espoirs suscités par la découverte de Harvey, que l'on songe par exemple à la manière dont Descartes en rend compte dans la $5^{\mathrm{e}}$ partie du Discours de la Méthode et comment on a pu penser à partir de là qu'une «mécanisation intégrale du corps machine» pourrait fournir à l'homme la possibilité de prolonger indéfiniment la vie ${ }^{65}$.

Il paraît tout aussi contestable de tirer parti de l'insistance mise par Erasistrate sur le peu d'impact thérapeutique de la découverte de Harvey pour en déduire l'incapacité de Fontenelle à concevoir l'importance de cette découverte du point de vue de la connaissance médicale et affirmer que les mathématiques seraient pour lui le seul domaine où s'est accomplie une véritable révolution ${ }^{66}$. Il ne s'agit pas tant pour lui de dire qu'il faudrait réserver

61 Sur ces échecs, voir Grmek 1990, 111. Voir aussi Journal des Savants, 31 janvier 1667, «Extrait du Journal d'Angleterre contenant la manière de faire passer le sang d'un animal à un autre», 31-36.

62 Salomon-Bayet 2008, 131-132.

63 «La nature veut que dans de certains temps, les hommes se succèdent les uns aux autres par le moyen de la mort; il leur est permis de se défendre contr'elle jusqu'à un certain point: mais passé cela, on aura beau faire de nouvelles découvertes dans l'anatomie, on aura beau pénétrer de plus en plus dans les secrets de la structure du corps humain, on ne prendra point la nature pour dupe; on mourra comme à l'ordinaire.» Fontenelle 1990, t. 1, 95.s

64 Mazauric 2007, 177, 208.

65 Même si ce projet d'une médecine infaillible suscité par les découvertes des anatomistes et des physiologistes et imputé à la philosophie cartésienne relève davantage d'une caricature de la pensée médicale et philosophique que de la réalité. Nous renvoyons sur ce point à l'article de D. Kolesnik: «Peut-on s'exempter de vieillir: l'apport cartésien» (paru dans Astérion, 8. 2011, [éds] «Vieillissement et prolongation de la vie», http://asterion.revues.org) qui souligne bien ce que cette vision du projet cartésien peut présenter de caricatural. Il faut aussi rappeler que Harvey lui-même s'est intéressé aux causes de la longévité, en proposant d'ailleurs une explication plus proche de la médecine ancienne (influence des régimes et des «six choses non naturelles») que de la médecine moderne. Voir à ce sujet Harvey 1847,587-592.

66 «Fontenelle could not conceive that this was a discovery of any great consequence for medicine.» «Finally, we would note that Fontenelle's assertion (through Erasistratus) that there will be no revolutions in medicine is the direct antithesis of Fontenelle's own recognition of the occurrence of a revolution in mathematics.» Cohen 1985, 88. 
l'usage du terme «révolution» aux sciences spéculatives, que de suggérer que ce terme n'est peut-être pas le plus adapté pour qualifier la découverte de Harvey.

C'est ce que tend à prouver la réponse faite par Harvey à Erasistrate lorsque celui-ci pointe l'inutilité de sa découverte. Certes, «découvrir un nouveau conduit dans le corps de l'homme» est tout aussi inutile que découvrir «une nouvelle étoile dans le Ciel». Pour autant, comme le souligne Harvey «il serait étrange qu'en connaissant mieux l'homme, on ne le guérit pas mieux» ${ }^{67}$. Autrement dit, les progrès thérapeutiques ne peuvent s'envisager indépendamment du projet d'une connaissance de l'homme. Si donc l'on peut parler d'une «supériorité» ou d'un «avantage» de la médecine moderne par rapport à la médecine ancienne, ce n'est pas en se fondant sur le critère de l'utilité (thérapeutique) mais sur celui d'une «refonte de l'homme», comme le fait remarquer le personnage de Harvey quelques lignes plus haut: «Depuis que j'ai eu trouvé une fois la circulation du sang [...] il semble qu'on ait refondu tout l'homme. Voyez combien notre médecine moderne doit avoir d'avantage sur la vôtre. Vous vous mêliez de guérir le corps humain, et le corps humain ne vous était seulement pas connu.» ${ }^{68}$

L'importance de la découverte de Harvey tient donc moins à la rupture qu'elle instituerait vis-à-vis de la science médicale des anciens, qu'à la manière dont elle engage une véritable refonte du savoir sur l'homme. Elle n'est peut-être pas à comprendre comme une découverte qui aurait permis à la médecine d'entrer, comme le disait Kant, «dans la voie sûre d'une science» ${ }^{69}$, une découverte à partir de laquelle le progrès des connaissances médicales pourrait s'envisager comme un processus continu. Elle constitue plutôt le point de départ d'une reconfiguration du savoir et des rapports entre connaissance (médicale) du corps d'un côté et connaissance (philosophique) de l'homme de l'autre.

Le terme de «refonte» apparaît alors plus adapté que celui de «découverte» ou de «révolution», car la mise au jour du principe de circulation sanguine ne signifie pas la fin de la médecine ancienne et du modèle humoriste de la santé et du corps, pas plus qu'elle n'instituerait le triomphe d'une conception purement mécaniste du corps. Son importance tient plutôt aux tensions, aux conflits qu'elle va susciter entre différents types de représentations du corps, à sa capacité à montrer les limites de chacun d'entre eux et la nécessité de s'engager, pour connaître le corps humain, dans une investigation sur le vivant en général (d'où l'importance de l'anatomie comparée

67 Fontenelle 1990, t. 1, 95 (nous soulignons).

68 Fontenelle 1990, t. 1, 93 (nous soulignons).

69 Kant 1990 [1787], 18. 
soulignée par Fontenelle: pour connaître l'homme il faut aussi étudier et connaître le corps des animaux).

Dans la Formation de l'esprit scientifique, Bachelard montre comment «les crises de croissance de la pensée impliquent une refonte totale du système du savoir ${ }^{70}$. Une rupture ne peut jamais se penser de manière purement isolée mais elle implique une réorganisation complète du savoir. Penser la discontinuité n'implique donc pas nécessairement de voir dans l'apparition d'une nouvelle théorie quelque chose qui impose le rejet des théories anciennes, mais plutôt de comprendre comment la nouveauté se traduit par une reconfiguration différente des savoirs anciens. Dès lors, l'ancienne théorie hippocratico-galénique des humeurs ne peut plus être envisagée comme un simple «obstacle épistémologique» que la découverte de la circulation sanguine permettrait enfin d'écarter. Il s'agit plutôt de comprendre comment cette théorie, admise pendant deux millénaires, se projette dans la nouvelle théorie (circulation sanguine) «sous la forme de tensions et de contradictions, les éléments conceptuels irréductibles aux anciennes conceptions commençant par y côtoyer les éléments conceptuels hérités de l'ancienne théorie» ${ }^{71}$.

C'est ce côtoiement et cette rencontre entre différents types de représentations du corps humain et de la santé humaine que Fontenelle donne à voir dans le cinquième de ses Dialogues des Morts et non une opposition tranchée entre anciennes et nouvelles théories. Erasistrate ne peut pas plus être considéré comme un «ancien» que Harvey ne peut revendiquer le statut de «moderne». Il s'agit aussi pour Fontenelle, dans son compte-rendu des débats autour de la circulation sanguine dans le fœetus, de montrer qu'aucun modèle physiologique n'est à même de rendre compte de manière systématique et définitive du fonctionnement du corps ou de l'origine de la vie. Voilà pourquoi une découverte médicale doit moins être évaluée du point de vue de ses usages pratiques (thérapeutiques), qu'en fonction de la manière dont elle nous aide à prendre conscience des limites du savoir médical et de la spécificité de son objet: le corps humain pensé comme un objet complexe et «assemblage de merveilles».

70 Bachelard 1989 [1938], 15-16.

71 Renault, article «Rupture» dans: Lecourt 2006, 982-83. 


\section{Bibliographie}

\section{Sources primaires}

Bernier, Jean, Essais de Médecine où il est traité de l'Histoire de la Médecine et des Médecins, du devoir des médecins à l'égard des malades et de celui des malades à l'égard des médecins. (Paris 1689)

Castle, George, The Chymical Galenist: A Treatise wherein the Practise of the Ancients is reconcil'd to the new Discoveries in the Theory of Physick (London 1667)

Descartes, René, CEuvres de Descartes: Discours de la Méthode et Essais, édité par C. Adam et P. Tannery, t. 6 (Paris 1996)

Diderot, Denis/Jean le Rond D'Alembert, Encyclopédie ou Dictionnaire raisonné des Sciences, des Arts et des Métiers, nouvelle impression en facsimilé de la première édition de 1751-1780, vol. 3, (Stuttgart/Bad Cannstatt 1988)

Dionis, Pierre, L'anatomie de l'homme suivant la circulation du sang, et les dernières découvertes. Démontrée au Jardin Royal. Par Mr. Dionis, premier chirurgien de Madame la Dauphine, Chirurgien ordinaire de la feuë Reine, \& Iuré à Paris (Paris 1690)

Fontenelle, Bernard le Bouyer de, Histoire de l'Académie Royale des Sciences avec les Mémoires de Mathématique et de Physique tirez des Registres de cette Académie (Paris, 1699-1703), http://gallica.bnf.fr/ark:/12148/bpt6k35013.r=histoire\%20acad\%C3\%A9mi\%20des\%20scie nces.langfr

Fontenelle, Bernard le Bouyer de, Nouveaux Dialogues des Morts, dialogue V, Euvres Complètes, t. 1 (Paris 1990)

Fontenelle, Bernard le Bouyer de, Entretiens sur la pluralité des mondes habités (1686), Euvres Complètes, t. 2 (Paris 1990)

Fontenelle, Bernard le Bouyer de, Préface sur l'utilité des mathématiques et de la physique, Euvres Complètes, t. 6 (Paris 1994)

Fontenelle, Bernard le Bouyer de, Éloges, Euvres Complètes, t. 7 (Paris 1996)

Fontenelle, Bernard le Bouyer de, Eléments de la géométrie de l'infini (1727), Préface de l'Histoire de l'Académie des sciences depuis 1666 jusqu'en 1699, Euvres complètes, t. 8 (Paris 2000)

Harvey, William, Anatomical examination of Thomas Parr, in: The Works of William Harvey (Londres 1847) 587-592

Harvey, William, Etude Anatomique du Mouvement du Caur et du Sang chez les Animaux, Aperçu historique et traduction française par Charles Laubry (Paris 1950)

Hobbes, Thomas, De Corpore, Elementorum Philosophiae Section Prima (Paris 1999 [1665])

Kant, Emmanuel, Critique de la Raison Pure, Préface à la Seconde édition (Paris 1990 [1787])

Merrett, Christopher, The Character of a Compleat Physician (Londres 1660)

Nedham, Marchamont, Medela Medicinae. A Plea for the Free Profession, and a Renovation of the Art of Physick. Out of the Noblest and most authentick Writers (Londres 1665)

Riolan, Jean, Manuel anatomique et pathologique ou abrégé de toute l'anatomie des usages que l'on peut en tirer pour la connaissance et pour la guérison des maladies (Paris 1652)

\section{Littérature secondaire}

Brian, Eric/Christiane Demeulenaere-Douyère (éds), Académie des sciences. Histoire et mémoire de l'Académie des sciences: Guide de recherches (Paris 1996)

Aucante, Vincent, La philosophie médicale de Descartes (Paris 2006)

Bachelard, Gaston, La formation de l'esprit scientifique (Paris 1989 [1938])

Bitbol-Hespériès, Annie, Le principe de vie chez Descartes (Paris 1990)

Boas Hass, Marie, The Scientific Renaissance, 1450-1630 (New York 1966 [1962])

Charbonneau,Frédéric, «Parallèles et symétries. Postérité du dialogue fontenellien», dans: MarcAndré Bernier (éd.), Parallèle des Anciens et des Modernes, Rhétorique, histoire et esthétique au siècle des Lumières (Laval 2006) 63-74

Cohen, I. Bernard, Revolution in Science (Cambridge/Londres 1985) 
Cook, Harold J. «The New Philosophy and Medicine in Seventeenth-Century England», in: David C. Lindberg/Robert S. Westman (eds), Reappraisals of the Scientific Revolution (Cambridge 2004 [1990]) 397-436

Cunningham, Andrew/Perry Williams, «De-Centring the 〈Big-Picture〉: ২The Origins of Modern Science> and the Modern Origins of Science», British Journal for the History of Science 26 (1993) 407-432, Allen, G. The English Paracelsians (New York 1965)

Franck, Robert J., «The Image of Harvey in Commonwealth and Restoration England», in: Jerome J. Bylebyl (ed.), William Harvey and His Age, The Professional and Social Context of the Discovery of the Circulation, The Johns Hopkins University Press (Baltimore/Londres 1979) 103-143

Franck, Robert J., Harvey and the Oxford Physiologists, A Study of Scientific Ideas (Berkeley/ Los Angeles/Londres 1980)

French, Roger, William Harvey's Natural Philosophy (Cambridge 1994)

French, Roger/Andrew Andrew, The Medical Revolution of the Seventeenth Century (Cambridge 1989)

Fuchs, Thomas, The Mechanization of the Heart: Harvey and Descartes (Rochester NY 2001)

Grmek, Mirko D., La première révolution biologique. Réflexions sur la physiologie et la médecine du XVII siècle (Paris 1990)

Grmek, Mirko D., Histoire de la pensée médicale en Occident, t. 2 (Paris 1997)

Hall, Rupert A., The Scientific Revolution, 1500-1800: The Formation of the Modern Scientific Attitude (Boston 1954 [1954])

Hall, Rupert A., From Galileo to Newton (New York 1963)

Hall, Rupert A., The Revolution in Science, 1500-1750 (Londres 1983)

Hunter, Michael, The Royal Society and its Fellows, 1660-1700: The Morphology of an Early Scientific Institution (Chalfont St Giles 1982)

Hunter, Michael, Establishing the New Science: The Experience of the Early Royal Society (Woodbridge 1989)

King, Lester S., «Attitudes towards «Scientific〉 Medicine around 1700», Bulletin of the History of Medicine 39 (1965) 124-133

Koyré, Alexandre, Du monde clos à l'univers infini (Paris 1990 [1957])

Kuhn, Thomas, La structure des révolutions scientifiques (Paris 2008 [1970])

Mazzolini, Renato G., «Les lumières de la raison: des systèmes médicaux à l'organologie naturaliste», dans: Mirko Grmek (éd.), Histoire de la Pensée Médicale en Occident, t. 2 (Paris 1997) 93-115

Mazauric, Simone, Fontenelle et l'invention de l'histoire des sciences à l'aube des Lumières (Paris 2007)

Osler, Margaret, Rethinking the Scientific Revolution (Cambridge 2000)

Pagel, Walter, William Harvey's Biological Ideas, Selected Aspects and Historical Background (Bâle 1967)

Renault, Emmanuel, «Rupture», dans: Dominique Lecourt (éd.), Dictionnaire d'histoire et philosophie des sciences (Paris 2006)

Salomon-Bayet, Claire, L'institution de la science et l'expérience du vivant (Paris 2008 [1978])

Seguin, Maria Susana, «Rhétorique scientifique et épistémologie dans les Mémoires de l'Académie des sciences», Revue Fontenelle 4 (2006) 147-159

Seguin, Maria Susana, «Du discours sur la nature au langage scientifique», Revue Fontenelle 6-7 (2010) 311-324

Shugg, Wallace/Walter Sherwin/Jay Freyman, «Henry More's <Circulatio Sanguinis»: an Unexamined Poem in Praise of Harvey», Bulletin of the History of Medicine 46 (1972) 180-189 